\title{
Myoelectric control techniques for a rehabilitation robot
}

\author{
Alan Smith and Edward E. Brown* \\ Biomechatronic Learning Lab, Department of Electrical and Microelectronic Engineering, Kate Gleason \\ College of Engineering, Rochester Institute of Technology, Rochester, NY, USA
}

\begin{abstract}
This work examines two different types of myoelectric control schemes for the purpose of rehabilitation robot applications. The first is a commonly used technique based on a Gaussian classifier. It is implemented in real time for healthy subjects in addition to a subject with Central Cord Syndrome (CCS). The myoelectric control scheme is used to control three degrees of freedom (DOF) on a robot manipulator which corresponded to the robot's elbow joint, wrist joint, and gripper. The classes of motion controlled include elbow flexion and extension, wrist pronation and supination, hand grasping and releasing, and rest. Healthy subjects were able to achieve $90 \%$ accuracy. Single DOF controllers were first tested on the subject with CCS and he achieved 100\%, 96\%, and 85\% accuracy for the elbow, gripper, and wrist controllers respectively. Secondly, he was able to control the three DOF controller at $68 \%$ accuracy. The potential applications for this scheme are rehabilitation and teleoperation. To overcome limitations in the pattern recognition based scheme, a second myoelectric control scheme is also presented which is trained using electromyographic (EMG) data derived from natural reaching motions in the sagittal plane. This second scheme is based on a time delayed neural network (TDNN) which has the ability to control multiple DOF at once. The controller tracked a subject's elbow and shoulder joints in the sagittal plane. Results showed an average error of $19^{\circ}$ for the two joints. This myoelectric control scheme has the potential of being used in the development of exoskeleton and orthotic rehabilitation applications.
\end{abstract}

Keywords: Electromyography, myoelectric control, rehabilitation robotics, assistive technologies

\section{Introduction}

The potential for robotics to play a vital role as an assistive technology for humans is increasing. It is predicted that senior citizens in the United States will double from forty million to eighty million by the year 2050 [2]. As a population ages, so does the amount of age related diseases and disorders such as stroke and Parkinson's disease. Robotics has the ability to help rehabilitate and improve the lives of these people. Cerebral palsy, multiples sclerosis, spinal cord injuries, and muscular dystrophy are other conditions in which robotic applications can also be developed to provide therapy and improve daily living [19]. In the field

*Corresponding author. E-mail: eebeee@ rit.edu. of rehabilitation robotics, robot and/or mechatronic technology is used to provide physically disabled people with the tools necessary to improve their quality of life and enhance their ability to operate in their environment [31]. A number of different applications in the field of rehabilitation robotics have been well established. Exoskeleton suits (which are wearable robotic/orthotic devices) have been created to augment human movement to help physically disabled people perform certain motions that they would otherwise not be able to perform [7, 25]. Other robotic devices have been built to provide physical therapy exercises for those suffering from stroke and other neuromuscular conditions in an attempt to rehabilitate them. Another category of applications include the teleoperation of a robotic device in a master slave relationship. 
Exoskeleton systems are attached directly to the body and typically assist and monitor limb data which includes EMG activity, kinematics, and forces. Guizzo and Goldstein [13] provide an overview of the latest exoskeleton systems being built and researched around the world. It includes the well known HAL (Hybrid Assistive Limb) robot from Japan and robots relating to the Defense Advanced Research Projects Agency (DARPA) efforts to name a few. Dollar and Herr [5] present a well written summary of autonomous exoskeletons and orthoses for the lower extremities that includes background terminology, history, present devices, and future research areas. Perry [30] and Gupta [14] have both presented well written developments of exoskeletons for the upper extremity and wrist respectively.

One of the first to incorporate robots into physical therapy was Hogan and Krebs at MIT [17]. Their robot, the MIT-Manus, has been used in the rehabilitation of stroke patients to improve joint mobility in the elbow, wrists, hands, shoulders, and ankles [20, 21]. Their work has shown the benefits of using robots in physical therapy over conventional methods. They have also reported positive results when using MANUS with those having cerebral palsy, multiple sclerosis, spinal cord injuries, and Parkinson's disease [19]. Song et al. [35] also developed a routine for robot aided arm training after stroke.

Essential to a rehabilitation robot application is the mode of interfacing the subject with the robot. Stiefelhagen et al. [36] explored many ways of interfacing with a robot. The interface of choice in this work is the electromyographic (EMG) signal. The use of the EMG signal as a source of control has been studied extensively in the field of prosthetics and is known as myoelectric control. Graupe et al. $[10,11]$ was one of the first to investigate potential uses of the EMG signal. A group at the University of New Brunswick have been studying and developing EMG prosthetic controllers for many years $[6,18]$. One of the most recent works in myoelectric control has been targeted muscle reinnervation (TMR) which has shown positive findings for those who have lost their upper extremity [22]. Oskoei and $\mathrm{Hu}$ [28, 29] compiled a well written survey on myoelectric control as well as performed their own work in myoelectric pattern classification.

Although relatively similar, a myoelectric control scheme for a rehabilitation robot application is different from a myoelectric control scheme for a prosthetic application. In a prosthetic application when there is a loss of a limb, one is forced to use EMG from muscles that remain after amputation. In a rehabilitation application, the goal is to utilize residual surface EMG information from a limb that is fully intact but does not have a fully functioning neuromuscular system. Some researchers have begun to integrate the EMG signal into rehabilitation applications. Gordon and Ferris [9] created an ankle exoskeleton which used the soleus muscle to derive a control signal. Fukuda et al. [8] developed a human assisting manipulator that was teleoperated using EMG signals to form a master-slave relationship. The physical therapy device mentioned previously and built by Song et al. [35] for the rehabilitation of stroke patients was based on myoelectric control, as well. Although there are some rehabilitation robot applications which incorporate myoelectric control, it is still unknown as to how much information the EMG signal can provide for rehabilitation purposes and in what form should that information be extracted. The goal of this work is to provide a framework upon which rehabilitation applications can be built using the EMG signal as the source of control.

The research focus of the Biomechatronic Learning Lab (BLL) at the Rochester Institute of Technology (RIT) is to develop intelligent orthotic and wearable robotic systems for those having neuromuscular diseases and disabilities. This work investigates uses of the surface EMG signal as a control input for a rehabilitation robotic application. It presents two methods of extracting control information from the EMG signal. The first method developed is based on a pattern recognition scheme that allows for control in three degrees of freedom (DOF), the elbow, wrist, and hand. This control scheme was developed in real time and is based on previously performed offline studies. Testing involved the use of healthy subjects and modifying the scheme for a subject having Central Cord Syndrome (CCS). This myoelectric control scheme incorporates muscles in the upper arm as well as muscles in the forearm. It also combines three degrees of freedom (elbow, wrist, and hand) in one complete control scheme. The second myoelectric control technique presented is based on trajectory tracking of joint angles. A control scheme based on actual joint and limb positions would offer benefits over the typical approaches based on pattern recognition and do not currently exist at this point in time. The proposed technique uses a time delayed neural network which allows for the control of multiple degrees of freedom instantaneously. The next section 
presents the theoretical background for both myoelectric control scheme methods. It also presents a small section on CCS. The third section presents the methodology and procedures followed for all of the tests with the results of the tests in the fourth section. The final two sections are the discussion and conclusion. The discussion analyzes the results and possible rehabilitation applications. The conclusion highlights the major points of the work and considers future work to be completed.

\section{Background}

\subsection{Myoelectric control scheme based on Gaussian classifier}

As mentioned earlier, there has been an extensive amount of research on EMG based control of prosthetic devices. The typical myoelectric control scheme used is based on pattern recognition theory. Figure 1

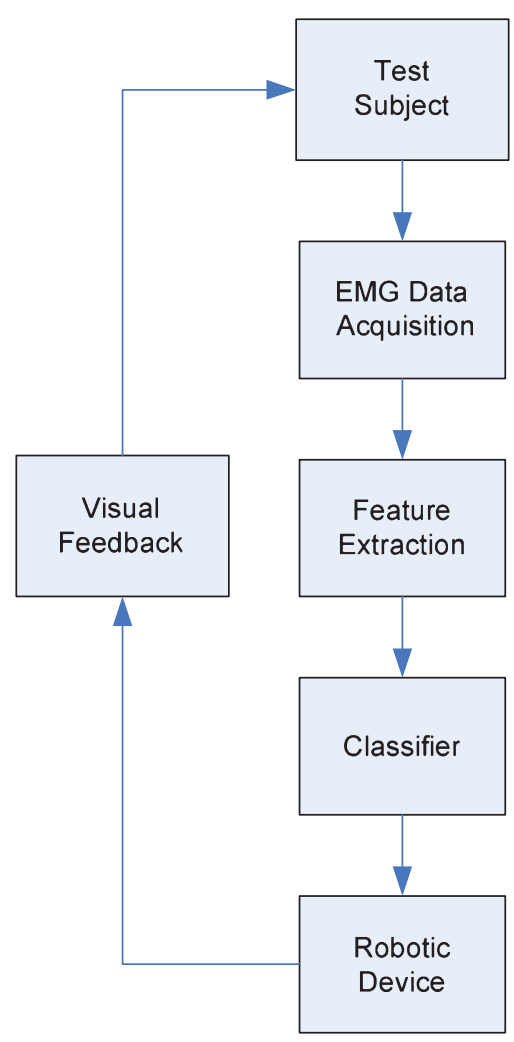

Fig. 1. Typical myoelectric control scheme based on pattern recognition. displays a block diagram that illustrates its implementation for a robotic device. For a system based purely on myoelectric control, all of the control inputs stem from EMG signals that are recorded from electrodes on the test subject's muscles. For a thorough understanding of EMG fundamentals, one is directed to [23]. The EMG data acquisition is typically obtained with sampling frequencies around $1 \mathrm{kHz}$ and filtered with a band-pass filter at $10-20 \mathrm{~Hz}$ to $400-450 \mathrm{~Hz}$. The data acquisition also includes any data segmenting or windowing techniques used. The heart of pattern recognition lies in the next two steps which are feature extraction and classification. Feature extraction involves using signal processing techniques to extract information from the EMG signals that discriminate between different types of muscle contractions or classes of motion such as elbow flexion or wrist pronation. Features can be broken into three different categories which include time domain, frequency domain, and time-frequency domain. These features are then passed to a classifier which makes a decision as to which class the features most likely belongs to. This approach does not predict specific reference angles or positions of joints and limbs, but rather it identifies a specific motion relative to the current position. The classifier output is then implemented on the robotic device being controlled. Lastly, the source of feedback in this myoelectric control scheme is the subject's visual feedback from seeing the movement of the robotic device. For more information about pattern recognition based myoelectric control schemes, one should consult Oskoei and $\mathrm{Hu}$ [28].

An initial offline analysis was performed to determine which windowing scheme and what features should be used in the real time system. The adjacent windowing technique is used in this work [18]. This technique uses a predefined window length and breaks the EMG signal into these lengths. In each of these adjacent segments, features are extracted and a class decision is made by the classifier which results in a processing delay. Typical windows range from $30 \mathrm{~ms}$ to $250 \mathrm{~ms}$. The EMG segment or window length should be large enough that the extracted features maintain consistency and the variance among samples are minimized, but one must note that a larger window results in a longer delay. Literature has established that the delay for a myoelectric control scheme should be no more than $300 \mathrm{~ms}$, so the choice of window size should account for this [28].

Five time domain features were tested in this work as described by Hudgins et al. [18]. These five time 
domain features were analyzed individually and collectively as a set (TD Set). They include the mean absolute value (MAV), the mean absolute value slope (MAVS), the zero crossings (ZC), the slope sign changes (SSC), and the waveform length (WL).

Autoregressive (AR) features were also investigated in this work. AR features are considered to be frequency features. Autoregressive features are obtained from an AR time-series model. As stated in the previous section, Graupe was one of the first to use AR features [10, 11]. An important consideration must be highlighted when considering the EMG signal as a time-series model. That is, that the EMG signal is nonlinear and nonstationary. This fact violates the assumptions made for a time-series model. Fortunately, the EMG signal can be modelled as a piece-wise stationary signal in smaller windows often used in myoelectric control. Graupe recommended using a fourth order model for estimating EMG signals. Hefftner et al. [16] also presented a lengthy analysis of using an AR model for the EMG signal. The AR model (with $m$ th order) is shown below in Equation 1 where the present EMG sample is $x_{k}$ and $w_{k}$ is white noise.

$$
x_{k}=\sum_{i=1}^{m} a_{i} x_{k-i}+w_{k}, \quad k=0,1,2 \ldots
$$

Two classifiers that are primarily used in the pattern recognition of EMG signals are Gaussian based classifiers and neural networks $[6,15,18,29]$. Both types of classifiers were explored in preliminary studies, but the Gaussian classifier consistently exhibited higher classification rates. As a result, the Gaussian classifier, which is sometimes termed a Bayesian classifier, was chosen for this work. A Gaussian classifier assumes that the features of each class belong to a Gaussian or normal distribution. To define a probability density function (PDF) for a normal distribution, all one needs are the mean vector and covariance matrix. In a Gaussian classifier, each class has a defined PDF which is found by extracting features from the training data of that class and calculating the mean vector and covariance matrix. Once the class PDFs are defined, features from an unknown EMG sample can be extracted and input into the PDF for each class. The unknown sample should be classified as belonging to the class that outputs the highest probability from the PDFs [38]. The probability density function (PDF) for the Gaussian classifier is defined in Equation 2 where $\mathrm{p}\left(x \mid \omega_{i}\right)$ is the probability of class $\omega_{i}$ given the feature vec- tor $x$ that has a mean vector, $\mu_{i}$, and a covariance matrix, $\sum_{i}$.

$$
\begin{aligned}
p\left(x \mid \omega_{i}\right) & =\frac{1}{(2 \pi)^{1 / 2}\left|\sum\right|_{i}^{1 / 2}} \exp \left(-\frac{1}{2}\left(x-\mu_{i}\right)^{T} \sum_{i}^{-1}\left(x-\mu_{i}\right)\right) \\
i & =1, \ldots, M
\end{aligned}
$$

\subsection{Myoelectric control scheme based on a time delayed neural network}

There are two drawbacks to a myoelectric control scheme based on the typical pattern recognition approach described above. The first drawback being that most myoelectric control systems are dependent on repeatable isometric contractions. A myoelectric control system based on transient muscle contractions derived from natural motions is highly desirable. The second limitation is that these systems are only able to control one DOF at a time. To overcome these limitations, a myoelectric control scheme based on a time delayed neural network (TDNN) is presented.

Several researchers have shown that it is possible to predict joint angular positions from the EMG signal for tracking applications $[3,37]$. The theory behind the TDNN presented here is largely based on previous work by $\mathrm{Au}$ and Kirsch [1], but their application was for functional neuromuscular stimulation. They discovered that the optimal parameters for implementing a TDNN for predicting joint position included a total delay of $875 \mathrm{~ms}$ along with a $125 \mathrm{~ms}$ interval of delay. This delay is too large for a myoelectric control application. This work assesses the TDNN as a potential architecture for a myoelectric control scheme. In order to verify the TDNN as an architecture for a myoelectric control scheme, the total delay was decreased while changing other TDNN variables. The accuracy of joint position for the TDNN is the measure for determining the optimal TDNN parameters.

A block diagram of the TDNN myoelectric control scheme is shown in Fig. 2. Raw EMG signals obtained from selected muscles are rectified and filtered prior to being input to the TDNN. The filter used is a lowpass butterworth filter located at $4 \mathrm{~Hz}$. The filtering is implemented because movements have no frequency content above this frequency. The EMG is not continuously fed into the TDNN. Rather, the EMG inputs are obtained by using an interval of delay, $\Delta t$, and a total delay, $n \Delta t$. That is, if the present time is $t$, then 

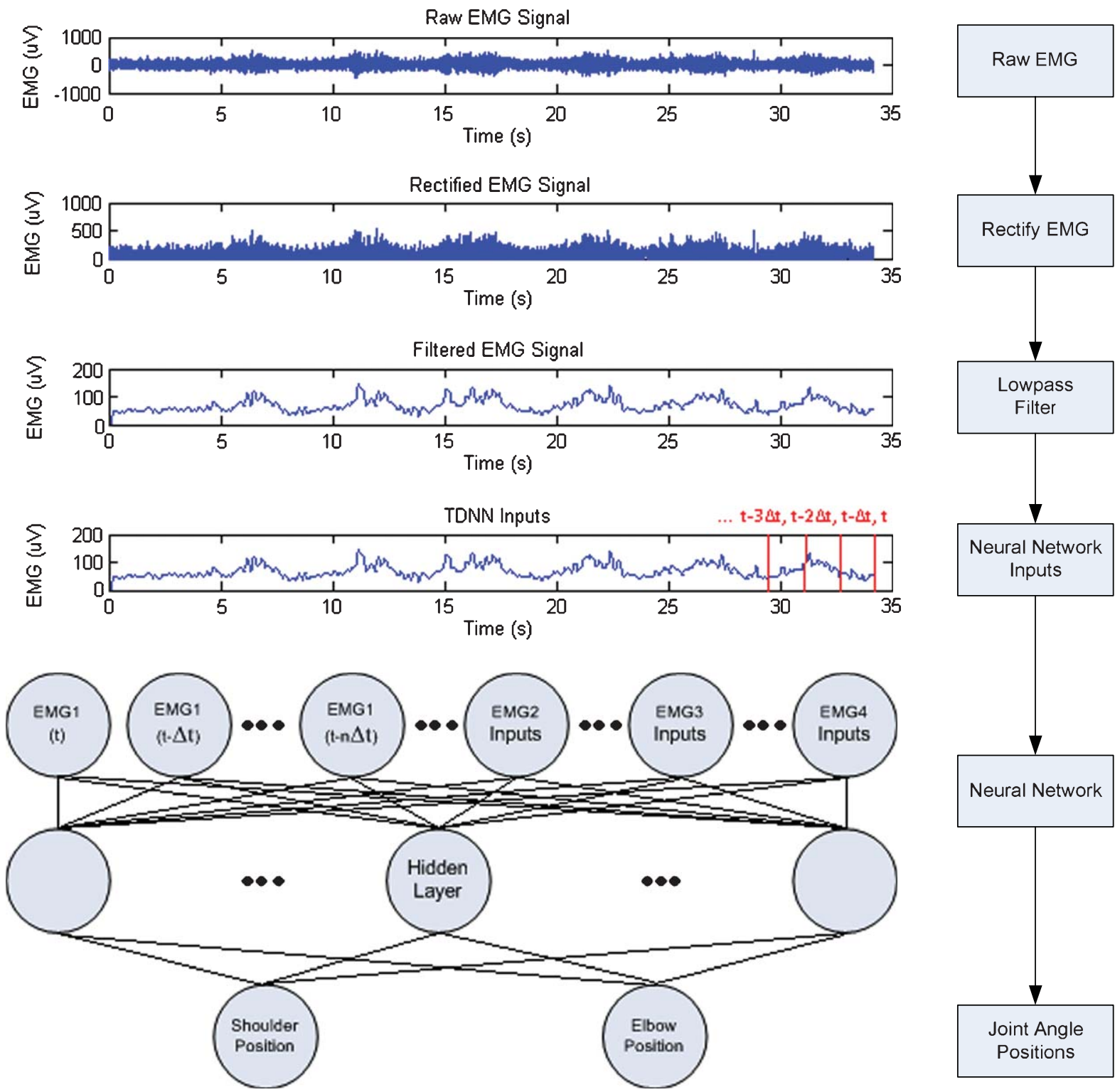

Fig. 2. Proposed joint tracking myoelectric control scheme using a TDNN.

the inputs of the TDNN would be the EMG values at $t, t-\Delta t, t-2 \Delta t$, and so on until one goes back in time to $n \Delta \mathrm{t}$. Before the TDNN can be used, it must be trained. Training data consisting of the EMG data with the coinciding joint positions must be used. During the training process the TDNN learns the mapping of the EMG inputs to the joint position outputs. Once trained the TDNN uses EMG to predict joint positions which can be used as commands for a controlled device.

\subsection{Central cord syndrome}

In myoelectric control systems, it is important to consider the end user of the control scheme. The majority of the time the designed myoelectric control system is not meant for a healthy person. And yet the majority of work in this area is used with healthy subjects. Typically the end user of a myoelectric control scheme is someone with a neuromuscular condition or an amputee. One of the goals of this work was to develop 
a foundational myoelectric control scheme for healthy subjects and then to extend that scheme to someone with a disability. Included in this work is the development of a myoelectric control scheme for someone with CCS.

CCS is the most common form of incomplete spinal cord injury. It is marked by disproportionately more motor loss and impairment in the upper limbs relative to the lower limbs. Other indicators of CCS include bladder dysfunction and varying degrees of sensory loss [39]. It is often seen in older patients with cervical spondylosis, which is a condition caused by abnormal wear on cartilage and the vertebrae. Older people with cervical spondylosis can experience injury due to hyperextension that results in pinching of the spinal cord [27]. Although CCS occurs most often in older persons, it can occur in those of any age brought on by a variety of injuries to the spinal cord [32]. Another condition sometimes associated with CCS is spasticity. Spasticity is an involuntary contraction of muscles which results from the nervous system sending signals to the muscles to contract although the person is not trying to contract $[4,34,40]$. It has been reported that those with CCS can have positive neurologic and functional recovery [12]. Although many people are able to regain some form of recovery, it is typically not a full recovery. Many CCS patients continue to suffer from neurological deficits that interfere with daily activities [41].

\section{Methodology}

\subsection{Myoelectric control scheme based on Gaussian classifier}

In order to initially test the real time three DOF myoelectric controller ten healthy subjects, five male and five female, were used. EMG channels were placed on the biceps, triceps, and four around the forearm. The four EMG channels from the forearm were evenly spread around the circumference of the forearm about one third the length of the forearm below the elbow. EMG data was collected at $960 \mathrm{~Hz}$ with the BioRadio 150 from Cleveland Medical Devices which filters the data from $0.5 \mathrm{~Hz}$ to $250 \mathrm{~Hz}$ [33]. An adjacent windowing scheme of $250 \mathrm{~ms}$ using AR features was implemented because the AR features outperformed the time domain features discussed earlier. Subjects were asked to produce isometric contractions that were approximately fifty percent of their maximum voluntary contraction to collect training data for each class (elbow flexion and extension, wrist pronation and supination, and hand grasping and releasing). Five seconds of EMG data were collected to train the classifier for each class. MATLAB Version 7.6 was used to extract the features from the training data and to calculate the mean vector and covariance matrix for each class.

The control scheme was implemented using Labview Version 8.5 and controlled the harmonically driven rehabilitation robot from Neuronics in Fig. 3 [26]. Once the system was trained, subjects practiced controlling the robot arm's elbow, wrist, and gripper joints. When the user felt confident in controlling each joint representing a specific motion class, the real time system was tested. If the subject could not adeptly control any of the motions, the system was retrained with new data. The real time test consisted of three different sets of randomized commands. Each set consisted of twenty-one commands that corresponded to three commands per motion class. The randomized sets of commands that were given to each subject can be seen in the results. Subjects were given a two minute rest period in between each set. The subjects attempted to execute the classes as they were commanded. If the subjects were able to hold the commanded class for three to four seconds, it was considered to be a correct classification. If subjects were unable to hold the command for three to four seconds, the command was considered to be misclassified. When a misclassification occurred, the output of the classifier was recorded. Classification accuracies were calculated as the number of successful classifications out of the total number of commands.

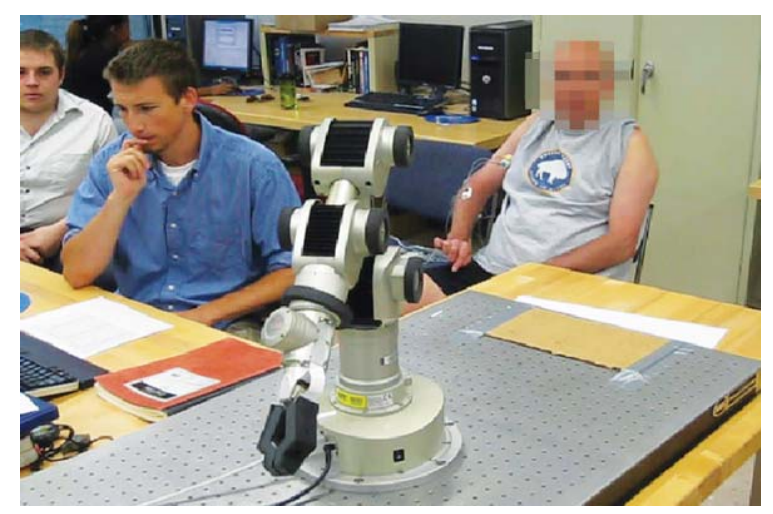

Fig. 3. CCS subject controlling the robotic arm from Neuronics. 
The myoelectric control scheme developed for the healthy subjects was applied to a subject with CCS. Due to the uncertainty of the subject's ability to control all three DOF at once, three different one DOF controllers were developed to test individually before testing the full controller. A controller for elbow flexion and extension was created using only EMG input from the biceps and triceps. Two separate controllers that used the four EMG channels on the forearm were also developed first for wrist pronation and supination, and, secondly, for hand grasping and releasing. The controllers were tested in a similar manner to the healthy subjects with three sets of randomized commands. Each set consisted of three commands per motion class which resulted in nine total commands per set. Once the CCS subject showed that he was able to proficiently control each DOF, the subject was tested using the full controller. Modifications were needed in order for the CCS subject to use the myoelectric controllers and will be explained in the discussion. The CCS subject is shown controlling the robotic arm in Fig. 3.

\subsection{Myoelectric control scheme based on a time delayed neural network}

All of the data presented in the work for the TDNN based myoelectric control scheme was collected using the Upper Extremity Motion Capture System shown in Figs 4 through 6 [24]. The developed system captures EMG and joint angle data from the elbow and shoulder. Data was obtained from 5 healthy subjects ( 4 males and 1 female) ranging in ages from 23 to 24 years of age. The first part of the work involved using the first subject's data to determine the optimal parameters for the TDNN. The rest of the subjects were then tested using the optimal TDNNs that used those parameters. EMG channels were placed on the biceps, triceps, deltoid, and pectoralis. The sampling rate for the data collection was $960 \mathrm{~Hz}$.

In order to make the TDNNs robust, several types of movement were collected. Single joint movements of both the elbow and the shoulder were collected. Single joint movement consisted of movement from rest over the full range of motion for the specified joint and back to rest. Rest was considered to be the arm located at the subject's side at full elbow extension. Full elbow extension was considered to be $180^{\circ}$ and full elbow flexion ranged from $50^{\circ}$ to $60^{\circ}$ depending on the subject as shown in Fig. 4. In the shoulder, the rest position was considered to be $0^{\circ}$ and the shoulder was elevated

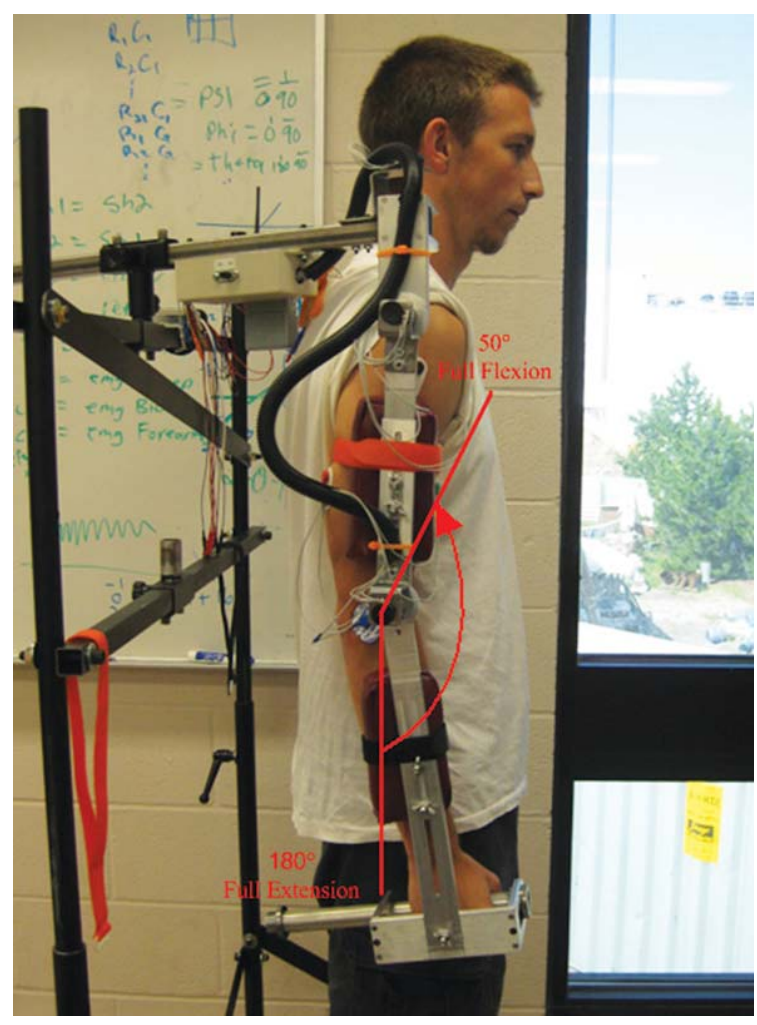

Fig. 4. Range of motion for single joint elbow motion.

to a position ranging between $90^{\circ}$ and $120^{\circ}$ depending on the subject as shown in Fig. 5. Reaching motions were also collected that resulted in the movement of both DOF at once as shown in Fig. 6. The reaching motions consisted of reaching toward different points in space from rest and then returning to rest. Each type of motion was recorded for slow and fast repe-

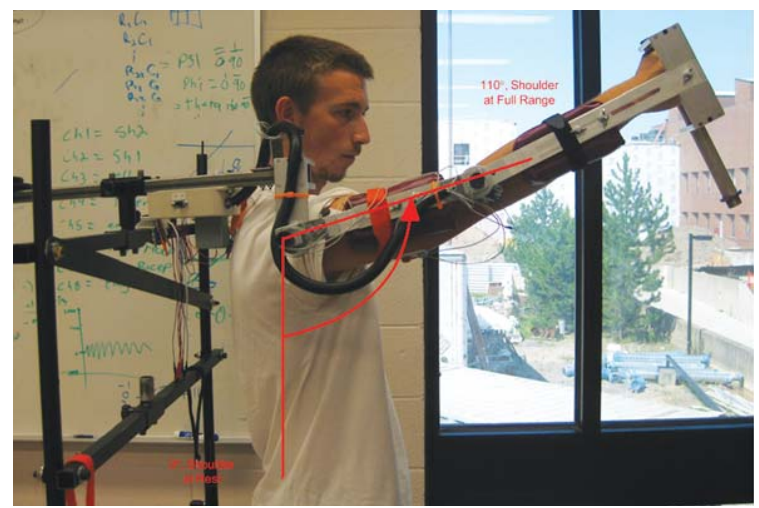

Fig. 5. Range of motion for single joint shoulder motion. 

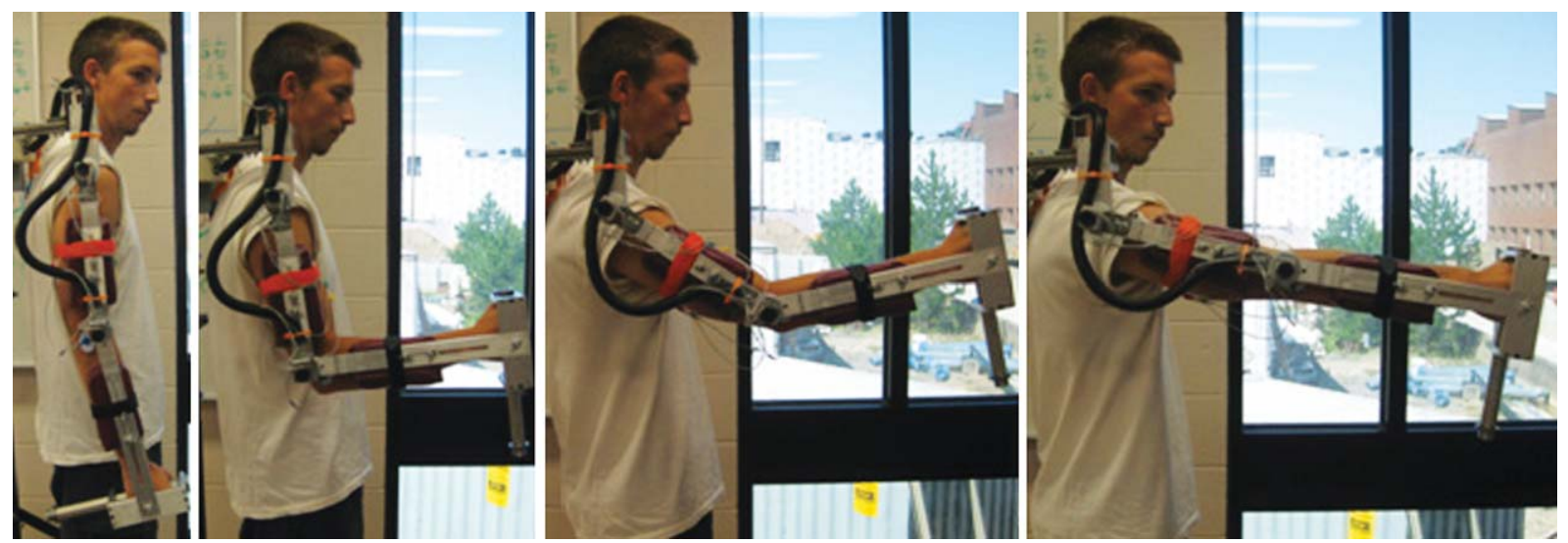

Fig. 6. Example of reaching motion involving motion in two degrees of freedom.

titions. Slow movements lasted for $3-4 \mathrm{~s}$ per repetition whereas fast movements lasted for $2-3 \mathrm{~s}$. In order to train the TDNNs for variability, the exact times were not constrained. Twelve different trials of data were collected with each trial lasting $30 \mathrm{~s}$ in length. Six trials were reserved for training, and six trials were used for testing the TDNN. The six trials consisted of elbow, shoulder, and reaching motions for both fast and slow movements.

The goal of the work with the TDNN was to investigate its possibility of using the TDNN output as a myoelectric control input by decreasing the time delay as stated as optimal in previous work. The time delays tested were $300 \mathrm{~ms}, 600 \mathrm{~ms}$, and $900 \mathrm{~ms}$. Other variables were also tested. The different delay intervals tested were $50 \mathrm{~ms}, 100 \mathrm{~ms}$, and $150 \mathrm{~ms}$. A single layer was used and the number of neurons tested was 10 , 20, 30, and 40 neurons. TDNNs were built for the first subject, and these corresponded to every possible combination of the parameters listed previously. The parameters that yielded the best results for Subject 1 were the parameters used to test the rest of the data for the other subjects. The use of the TDNN was a two step process consisting of training and testing. As recommend by Au and Kirsch [1], the position data was normalized between 0 and 1 . All of the neural network simulations were executed using MATLAB's neural network toolbox, Version 7.6. The neural network created was a feed-forward, back propagation network. The "tansig transfer function" was used for the hidden layer and a "linear transfer function" was used for the output layer. The training was limited to a maximum of 250 iterations.

\section{Results}

\subsection{Myoelectric control scheme based on Gaussian classifier}

A sample of an individual's results is shown in Table 1 for the first female subject. It shows the randomized set of commands for the three sets and the result of each command. Additionally, it shows the commands that were correctly classified as well as the output of the classifier during any misclassifications. The classification results of testing all 10 healthy subjects are shown in Fig. 7. In order to gain an understanding of what classes were misclassified; one is directed to Fig. 8. Figure 8 shows the percentage of each class that was misclassified out of the total number of misclassifications for all subjects. The real time test results for the three single DOF myoelectric controllers and the full three DOF myoelectric controllers developed for the subject with CCS are shown in Fig. 9. Figure 10 shows the percentage of each class that was misclassified out of the total number of misclassifications for the full controller for the CCS subject.

\subsection{Myoelectric control scheme based on a time delayed neural network}

By varying all of the TDNN parameters, 36 different neural networks were created for the first subject. Each neural network was trained using the six designated training trials of movement. Once trained, the neural networks were then tested with the six trials of test data. The results showed that varying the number 
Table 1

Randomized commands given to all subjects for testing of the real time myoelectric control scheme and the results for the first female subject

\begin{tabular}{|c|c|c|c|c|c|c|c|c|c|}
\hline \multirow[t]{3}{*}{ Command } & \multicolumn{9}{|c|}{ Female Subject 1} \\
\hline & \multicolumn{3}{|c|}{ Set 1} & \multicolumn{3}{|c|}{ Set 2} & \multicolumn{3}{|c|}{ Set 3} \\
\hline & Class & Classified & Misclassified & Class & Classified & Misclassified & Class & Classified & Misclassified \\
\hline 1 & Flexion & $\mathrm{X}$ & & Grasping & $\mathrm{X}$ & & Extension & $\mathrm{X}$ & \\
\hline 2 & Pronation & $\mathrm{X}$ & & Pronation & $X$ & & Flexion & $\mathrm{X}$ & \\
\hline 3 & Grasping & $X$ & & Releasing & $X$ & & Rest & & Sup/Ext \\
\hline 4 & Pronation & $X$ & & Extension & & Sup/Rel & Grasping & $X$ & \\
\hline 5 & Rest & $\mathrm{X}$ & & Releasing & $X$ & & Releasing & $\mathrm{X}$ & \\
\hline 6 & Grasping & $\mathrm{X}$ & & Pronation & $\mathrm{X}$ & & Supination & $\mathrm{X}$ & \\
\hline 7 & Extension & & Releasing & Rest & $\mathrm{X}$ & & Rest & $\mathrm{X}$ & \\
\hline 8 & Flexion & $X$ & & Extension & $\mathrm{X}$ & & Flexion & $\mathrm{X}$ & \\
\hline 9 & Extension & $\mathrm{X}$ & & Grasping & $\mathrm{X}$ & & Supination & $\mathrm{X}$ & \\
\hline 10 & Supination & $X$ & & Flexion & $X$ & & Pronation & $\mathrm{X}$ & \\
\hline 11 & Grasping & $X$ & & Supination & $X$ & & Extension & $\mathrm{X}$ & \\
\hline 12 & Releasing & $\mathrm{X}$ & & Releasing & $\mathrm{X}$ & & Flexion & $\mathrm{X}$ & \\
\hline 13 & Flexion & $X$ & & Pronation & $X$ & & Releasing & $\mathrm{X}$ & \\
\hline 14 & Grasping & $\mathrm{X}$ & & Supination & $\mathrm{X}$ & & Supination & $\mathrm{X}$ & \\
\hline 15 & Rest & & Ex/Flex & Rest & $\mathrm{X}$ & & Rest & $\mathrm{X}$ & \\
\hline 16 & Extension & $\mathrm{X}$ & & Supination & $\mathrm{X}$ & & Pronation & $\mathrm{X}$ & \\
\hline 17 & Supination & $\mathrm{X}$ & & Flexion & $\mathrm{X}$ & & Grasping & $\mathrm{X}$ & \\
\hline 18 & Releasing & $\mathrm{X}$ & & Extension & $\mathrm{X}$ & & Extension & & Gra/Pro \\
\hline 19 & Pronation & $\mathrm{X}$ & & Rest & $\mathrm{X}$ & & Releasing & $\mathrm{X}$ & \\
\hline 20 & Rest & $\mathrm{X}$ & & Grasping & $\mathrm{X}$ & 0 & Pronation & $\mathrm{X}$ & \\
\hline 21 & Supination & $\mathrm{X}$ & & Flexion & $\mathrm{X}$ & & Grasping & $\mathrm{X}$ & \\
\hline \multicolumn{2}{|c|}{ Classification accuracy } & & $90.48 \%$ & & & $95.24 \%$ & & & $90.48 \%$ \\
\hline \multicolumn{2}{|c|}{ Total accuracy } & & & & & & & & $92.06 \%$ \\
\hline
\end{tabular}

Real Time Classification Accuracies

for All Subjects

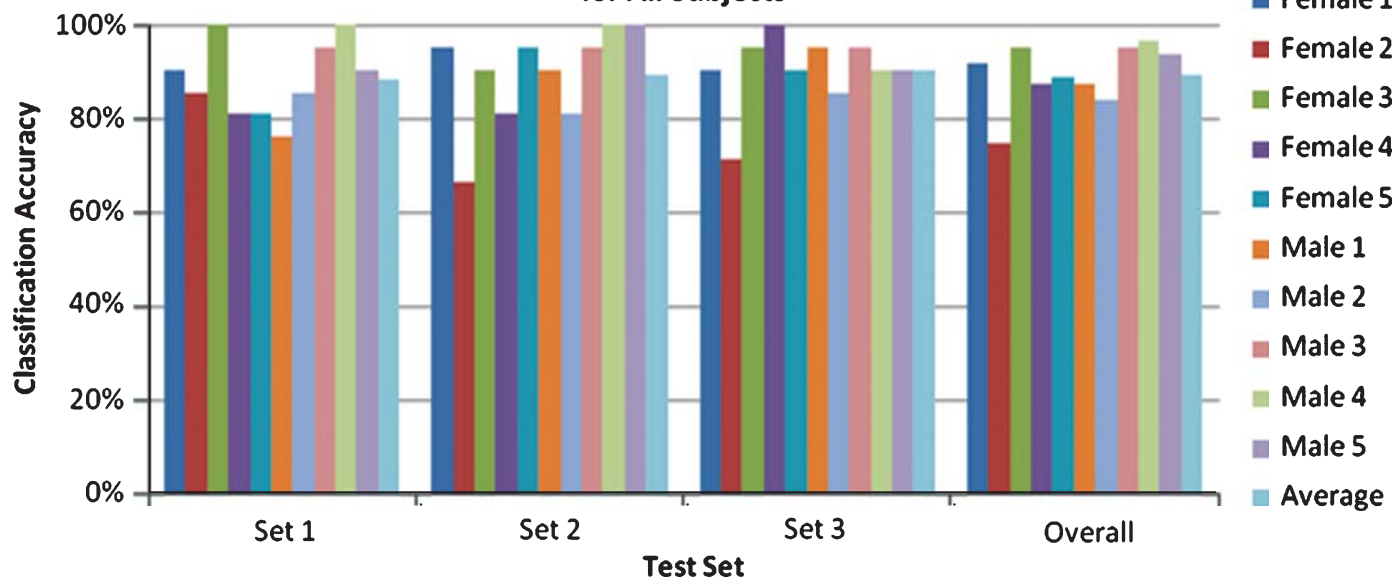

Fig. 7. Real time test results for all of the healthy subjects shown as individual test sets and overall averages.

of neurons in the TDNN did not result in a change of accuracy. Therefore, it has been chosen to only show the results corresponding to the TDNNs with 10 neurons in the hidden layer. Table 2 displays the combined average error for the shoulder and elbow combined for Subject 1 while varying the total delay and the delay interval. Table 3 presents a more detailed breakdown of the errors for each specific motion and each joint over the different TDNNs for Subject 1. For simplicity in presenting the results, only the detailed results for the TDNNs having $100 \mathrm{~ms}$ as the delay interval are shown in Table 3. This was chosen due to the fact that the $100 \mathrm{~ms}$ delay interval performed slightly better than the $50 \mathrm{~ms}$ and $150 \mathrm{~ms}$ intervals as seen in Table 2. 


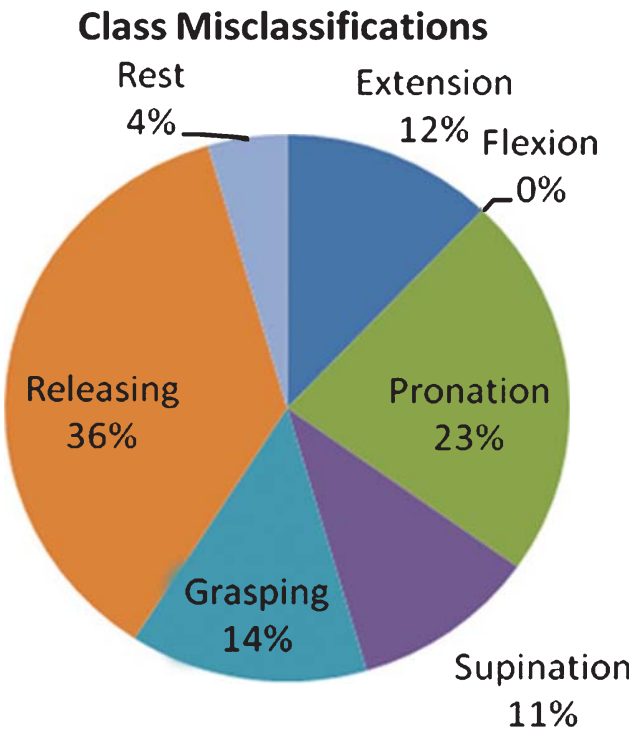

Fig. 8. Percentage of each individual class that was misclassified out of the total number of misclassifications for all subjects.

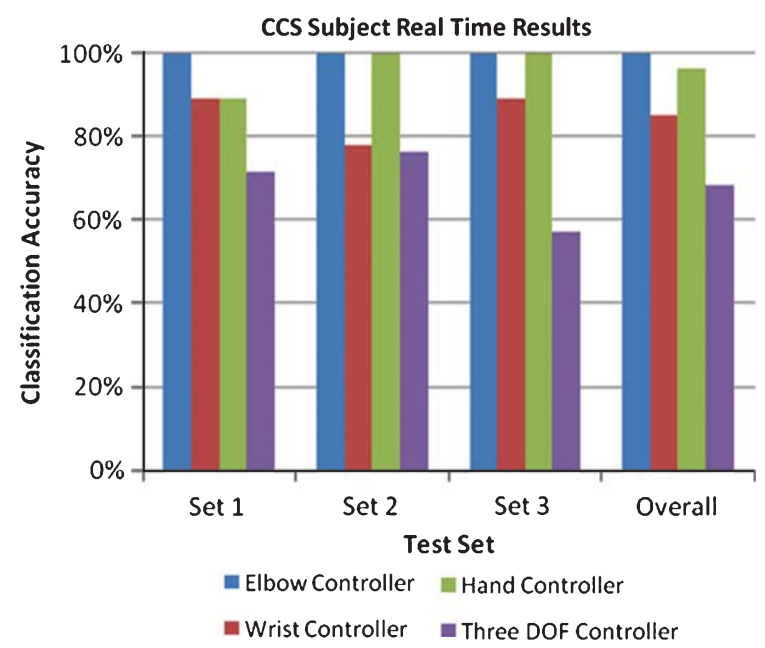

Fig. 9. Real time test results of all four controllers for the CCS subject shown as individual test sets and overall averages.

Displayed in Table 4 is the average error of each DOF for each subject during all test trials. The results in Table 4 are for TDNNs having a time delay of $300 \mathrm{~ms}$, a time delay interval of $100 \mathrm{~ms}$, and 10 neurons, parameters determined by the results of Subject 1 . Table 5 displays the average error of the shoulder and elbow combined for all the subjects using a TDNN. All errors are presented with a standard deviation. A key feature of the suggested TDNN control approach is the ability to track more than $1 \mathrm{DOF}$ at a time. Two test trials

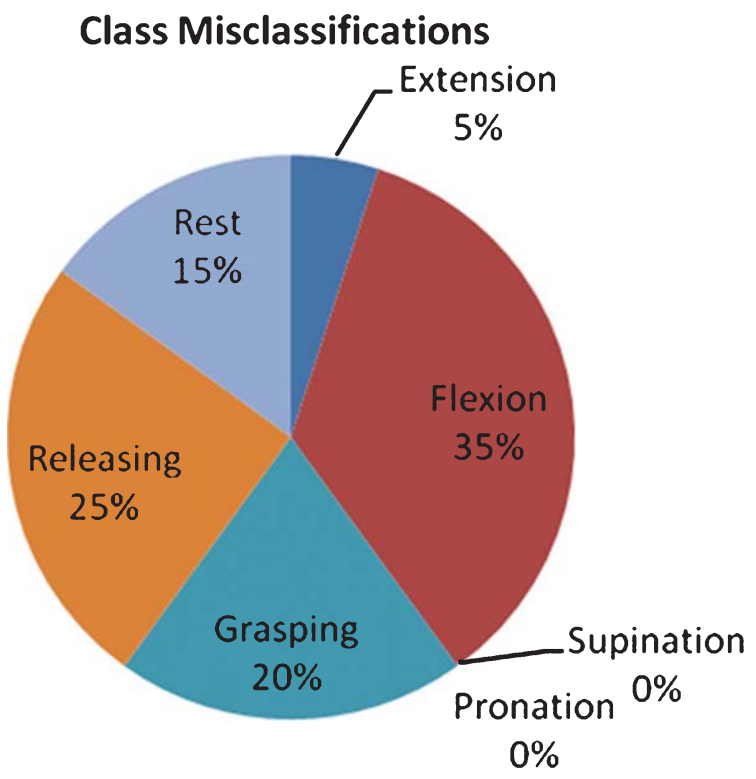

Fig. 10. Percentage of each individual class that was misclassified out of the total number of misclassifications for the CCS subject.

Table 2

The overall error for each TDNN neural network with 10 neurons in the hidden layer while varying time delay and time interval for Subject 1

\begin{tabular}{lcc}
\hline \multicolumn{3}{c}{ Overall TDNN errors (degrees) } \\
\hline Time delay & Time interval & Average error \\
\hline 300 & 50 & $16.0 \pm 23.2$ \\
300 & 100 & $15.7 \pm 22.9$ \\
300 & 150 & $15.7 \pm 22.8$ \\
600 & 50 & $17.1 \pm 24.2$ \\
600 & 100 & $16.3 \pm 23.8$ \\
600 & 150 & $16.5 \pm 23.5$ \\
900 & 50 & $17.3 \pm 23.5$ \\
900 & 100 & $16.9 \pm 23.7$ \\
900 & 150 & $22.8 \pm 30.5$ \\
\hline
\end{tabular}

of the TDNN having a delay of $300 \mathrm{~ms}$ and intervals of $100 \mathrm{~ms}$ are displayed in Figs 11 and 12. Figure 11 shows the output of the TDNN for fast shoulder movement by Subject 2. Figure 12 shows the output for a slow reaching movement by Subject 1 .

\section{Discussion}

\subsection{Pattern recognition myoelectric control scheme}

The majority of myoelectric pattern classification publications perform offline studies that have 
Table 3

The error of shoulder position and elbow position for Subject 1 for each type of movement. The TDNNs shown here had 10 neurons in the hidden layer and a delay interval of $100 \mathrm{~ms}$.

\begin{tabular}{|c|c|c|c|c|c|c|c|}
\hline Time delay & Time interval & Slow elbow & Fast elbow & Slow shoulder & Fast shoulder & Slow reaching & Fast reaching \\
\hline \multicolumn{8}{|c|}{ Shoulder error for Subject 1 (degrees) } \\
\hline 300 & 100 & $6.0 \pm 6.3$ & $7.5 \pm 10.2$ & $16.4 \pm 19.7$ & $13.2 \pm 17.0$ & $11.2 \pm 15.1$ & $14.5 \pm 21.4$ \\
\hline 600 & 100 & $7.0 \pm 7.6$ & $9.0 \pm 11.5$ & $15.2 \pm 19.0$ & $15.3 \pm 19.0$ & $11.0 \pm 15.8$ & $14.2 \pm 18.7$ \\
\hline 900 & 100 & $6.0 \pm 8.3$ & $9.8 \pm 12.7$ & $14.9 \pm 18.6$ & $13.2 \pm 17.4$ & $10.9 \pm 15.8$ & $16.8 \pm 24.6$ \\
\hline \multicolumn{8}{|c|}{ Elbow error for Subject 1 (degrees) } \\
\hline 300 & 100 & $25.9 \pm 33.5$ & $21.7 \pm 28.8$ & $20.4 \pm 19.6$ & $18.2 \pm 18.6$ & $16.1 \pm 20.6$ & $17.7 \pm 24.9$ \\
\hline 600 & 100 & $25.3 \pm 32.1$ & $22.0 \pm 28.3$ & $20.7 \pm 20.3$ & $18.6 \pm 22.1$ & $17.4 \pm 25.7$ & $20.4 \pm 27.6$ \\
\hline 900 & 100 & $29.7 \pm 36.9$ & $24.3 \pm 27.0$ & $17.3 \pm 12.2$ & $18.6 \pm 13.3$ & $17.6 \pm 20.9$ & $23.9 \pm 31.1$ \\
\hline
\end{tabular}

Table 4

The error of shoulder and elbow position over each movement for each subject. Each TDNN had 10 neurons in the hidden layer with a delay interval of $100 \mathrm{~ms}$.

\begin{tabular}{|c|c|c|c|c|c|c|}
\hline Test subject & Slow elbow & Fast elbow & Slow shoulder & Fast shoulder & Slow reaching & Fast reaching \\
\hline \multicolumn{7}{|c|}{ Shoulder error (degrees) } \\
\hline 1 & $6.0 \pm 6.3$ & $7.5 \pm 10.2$ & $16.4 \pm 19.7$ & $13.2 \pm 17.0$ & $11.2 \pm 15.1$ & $14.5 \pm 21.4$ \\
\hline 2 & $19.1 \pm 24.1$ & $21.2 \pm 25.7$ & $12.0 \pm 10.8$ & $21.6 \pm 13.3$ & $20.3 \pm 27.4$ & $20.1 \pm 26.3$ \\
\hline 3 & $6.4 \pm 8.2$ & $11.0 \pm 14.6$ & $27.7 \pm 27.0$ & $27.6 \pm 33.5$ & $20.1 \pm 24.6$ & $19.7 \pm 26.2$ \\
\hline 4 & $15.2 \pm 24.9$ & $19.2 \pm 27.8$ & $26.1 \pm 36.9$ & $29.8 \pm 41.9$ & $29.6 \pm 39.6$ & $35.6 \pm 43.4$ \\
\hline 5 & $4.2 \pm 5.1$ & $6.9 \pm 10.5$ & $11.6 \pm 12.6$ & $12.1 \pm 15.8$ & $14.6 \pm 17.6$ & $15.9 \pm 19.1$ \\
\hline \multicolumn{7}{|c|}{ Elbow error (degrees) } \\
\hline 1 & $25.9 \pm 33.5$ & $21.7 \pm 28.8$ & $20.4 \pm 19.6$ & $18.2 \pm 18.6$ & $16.1 \pm 20.6$ & $17.7 \pm 24.9$ \\
\hline 2 & $6.5 \pm 9.2$ & $10.3 \pm 15.3$ & $13.7 \pm 16.4$ & $18.4 \pm 23.2$ & $18.8 \pm 24.9$ & $22.5 \pm 25.5$ \\
\hline 3 & $34.3 \pm 40.0$ & $29.1 \pm 33.6$ & $26.8 \pm 21.0$ & $30.1 \pm 22.8$ & $23.3 \pm 28.1$ & $27.6 \pm 32.7$ \\
\hline 4 & $28.4 \pm 29.9$ & $22.8 \pm 30.6$ & $10.4 \pm 10.2$ & $16.0 \pm 14.5$ & $11.5 \pm 15.0$ & $13.3 \pm 17.6$ \\
\hline 5 & $21.2 \pm 24.7$ & $31.8 \pm 36.3$ & $17.8 \pm 23.5$ & $22.3 \pm 23.6$ & $29.6 \pm 35.2$ & $21.0 \pm 26.8$ \\
\hline
\end{tabular}

Table 5

The average error of the shoulder and elbow position combined for all movements for a TDNN having 10 neurons in the hidden layer with a delay interval of $100 \mathrm{~ms}$.

\begin{tabular}{lc}
\hline Test subject & Average error \\
\hline 1 & $15.7 \pm 22.9$ \\
2 & $17.0 \pm 23.2$ \\
3 & $23.6 \pm 30.6$ \\
4 & $21.5 \pm 31.4$ \\
5 & $17.4 \pm 25.3$ \\
\hline
\end{tabular}

extremely tight constraints on their data collection. Many researchers use a device that holds the arm stationary which helps to ensure the EMG data is isometric. These constraints allow for a higher rate of classification, but they cannot be recreated in a real time system for an actual rehabilitation application. It is essential for work in pattern recognition of EMG signals to go beyond offline studies. Offline studies do not incorporate any visual feedback for the user. Feedback for control systems is essential. In noninvasive myoelectric control systems the only feedback currently available is visual feedback from the device being con- trolled. Without feedback, an analysis of EMG pattern classification is incomplete. The myoelectric control scheme developed here was evaluated in real time for both healthy subjects and an end user who could potentially benefit from a myoelectric control scheme.

The real time myoelectric control scheme was first tested on healthy subjects and the results are shown in Fig. 7. After training the system, the subjects were allowed to control the robotic arm incorporating visual feedback to the control scheme. Some subjects required a second set of training data to be collected in order for the classifier to accurately identify all the classes. Once the subjects felt comfortable controlling the robotic arm, they were tested using the three sets of randomized commands. The average classification rate for all of the healthy subjects was $89.52 \%$. Male Subject 4 had the highest classification accuracy with $96.83 \%$ and this corresponded to two misclassifications out of the sixty-three total commands given to the subject over testing. The worst classification occurred for female Subject 2 who had a classification accuracy of $74.60 \%$. This subject noted that she became fatigued, which is seen in the drop of her classification 

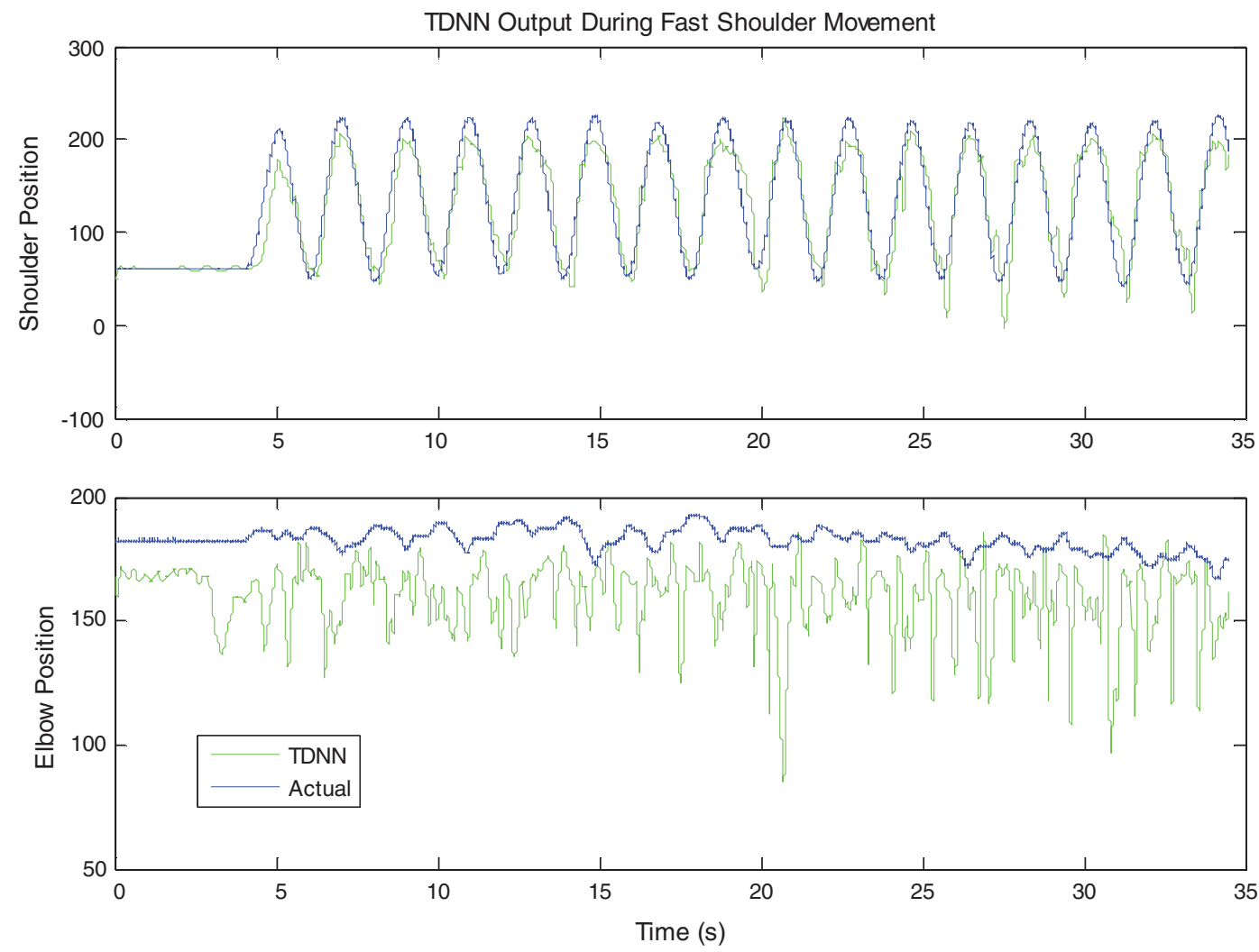

Fig. 11. The output of the TDNN during fast shoulder movement for Subject 2.

accuracy after the first set. A look at her misclassifications for the last two sets showed that the majority of misclassifications were occurring for the pronation, supination, and releasing classes. The majority of the outputs from the controller during these misclassifications were extension which meant that fatigue was causing the triceps to contract. This resulted in extension being the output from the classifier. If one were to exclude female Subject 2 from the results because of her fatigue, the average accuracy for the tests would be $91.18 \%$.

In order to analyze the misclassifications that occurred during the real time tests, Fig. 8 was created. This shows which classes were misclassified most often as well as the number of class misclassifications relative to each other. Releasing and pronation made up for $36 \%$ and $23 \%$ of misclassifications. Both of these movements are derived from the forearm muscles and the higher misclassification rates could be a result of there being four classes of motion stemming from the forearm EMG channels (pronation, supination, grasp- ing, releasing) versus one class each from the biceps (flexion) and triceps (extension). This could also be the significance of why there were no misclassifications for flexion. The biceps is primarily involved in one motion which is flexion while that is not the case for the forearm where so many muscles are located in close proximity.

Crucial to the evaluation of a myoelectric control scheme is the inclusion of end users as test subjects. The tests for the healthy subjects were intended to create a foundational myoelectric control system which could be adapted for a person with CCS. To the authors' knowledge no attempts have been made to incorporate a myoelectric control scheme for a subject with CCS. The testing of the subject with CCS took place over several visitations to the BLL at RIT on account of necessary adaptations that needed to be made to the control scheme. Because of the uncertainty of the CCS subject's ability to control all three DOF using the control scheme the healthy subjects used, three single DOF controllers were developed that were less complex. It 

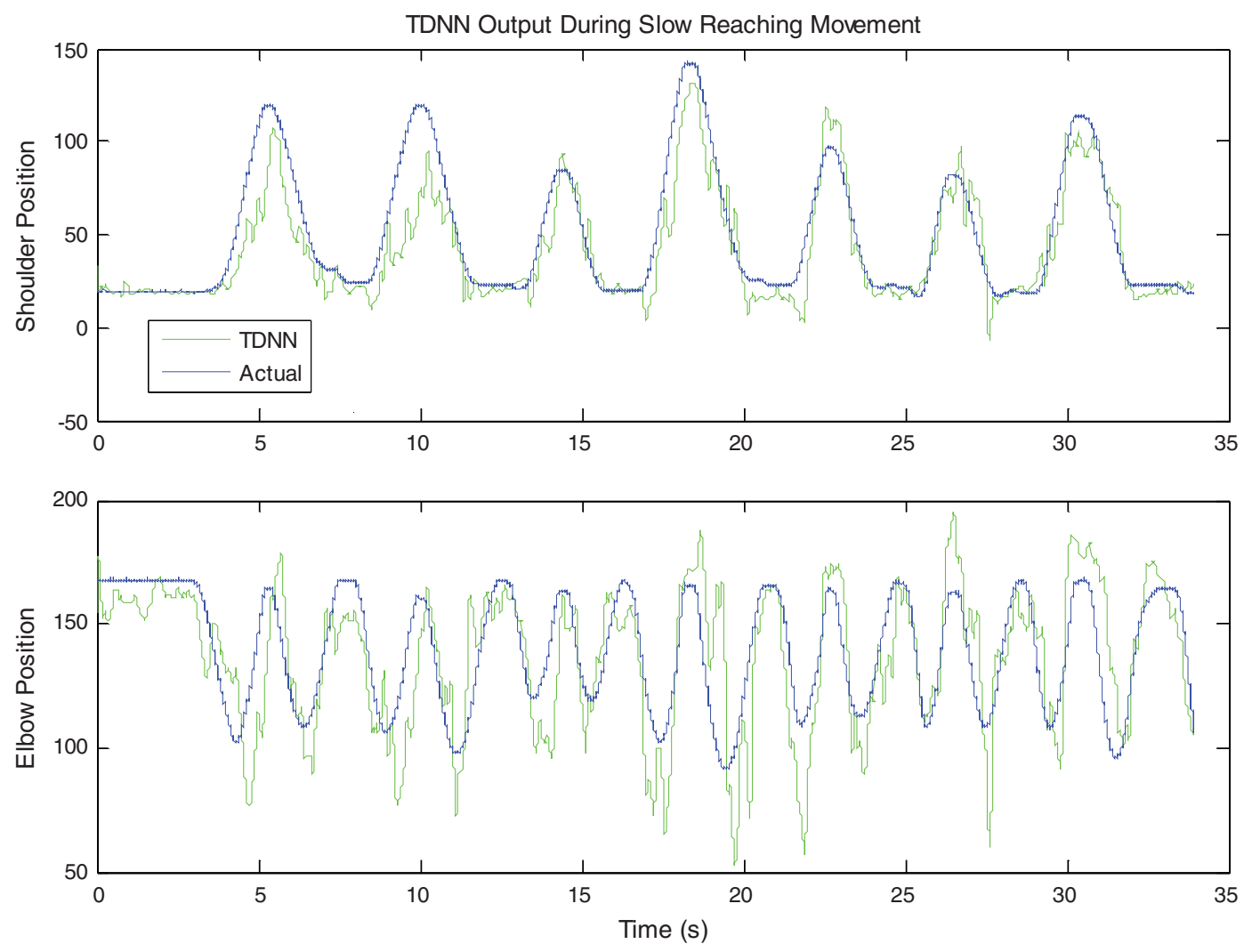

Fig. 12. The output of the TDNN during slow reaching movement for Subject 1.

was the goal of the authors to establish a series of successful trials for the subject using the simpler controllers in order to establish the subject's confidence in myoelectric control. Each single DOF controller was tested separately. The first controller was for the elbow joint which used the biceps and triceps EMG as inputs. The subject was able to use this controller with $100 \%$ accuracy as shown in Fig. 9. For the wrist controller, the subject was unable to have success in controlling the joint using the four forearm channels. After viewing the subject's attempt at performing wrist pronation and supination, it was noticed that he had a difficult time and compensated by using his biceps. By changing the control scheme to incorporate a biceps EMG channel, the subject was able to use the controller successfully and achieved $85.19 \%$ overall accuracy. The subject used the four forearm inputs to effectively control the hand controller for releasing and grasping. The accuracy for the hand controller was $96.30 \%$.

Use of the full three DOF controller was attempted following success with the individual controllers. Sev- eral attempts were made at training the three DOF controller, but the subject was unable to achieve any level of success. Again, it was noticed that the subject overcompensated to perform the arm motions with his shoulder muscles. It was then decided to use two forearm channels on the extensors and flexors. The other two forearm channels were moved to the shoulder as shown in Fig. 13. The shoulder EMG channels were placed on the deltoid and on the trapezius muscles. Once these locations were used, the subject could be tested using the three sets of randomized commands. The subject had an overall classification accuracy of $68.25 \%$ for the three DOF controller which is lower than the classification rates for the single DOF controllers and the results for the healthy subjects. This can be attributed to a number of factors. The first factor is that muscle spasticity set into his arm muscles as talked about in the background section on CCS. This caused involuntary muscle contractions and was due to the higher number of commands in the tests for the three DOF controller versus the single DOF 


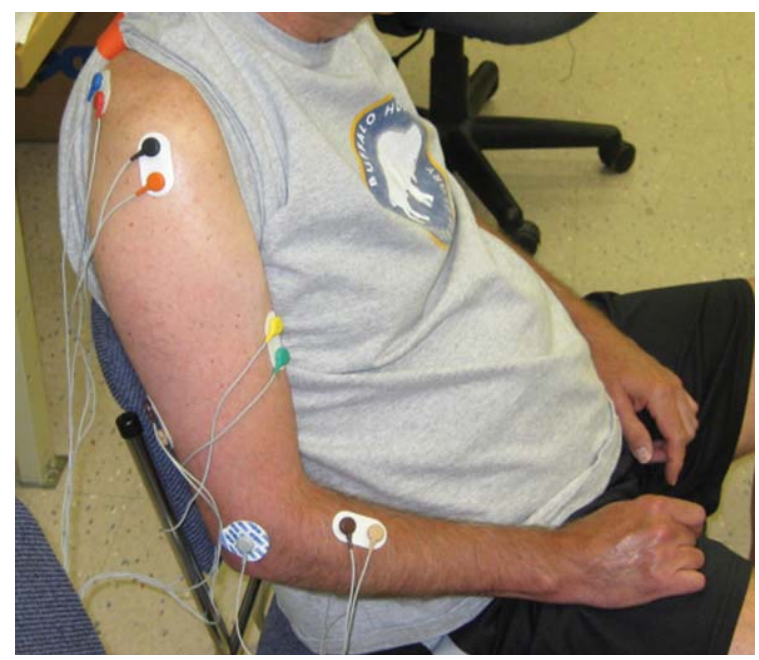

Fig. 13. Displayed here is the EMG channel placement for the CCS subject for the three DOF myoelectric control scheme which includes wrist extensors and flexors, biceps, triceps, deltoid, and trapezius.

controllers. The second factor is due to the compensatory use of muscles. For instance the biceps was used to compensate for a lack of wrist strength during wrist pronation and supination. This is shown in Fig. 10 which shows a large amount of misclassification for flexion. This is due to the contraction of the biceps being classified as pronation and supination. The importance of incorporating end users into tests of a myoelectric control scheme is shown in the difference of Fig. 10 for the CCS subject and Fig. 8 for healthy subjects. Where flexion had a perfect classification rate for the healthy subjects, the CCS subject had the greatest difficulty with flexion. An understanding of a myoelectric control scheme is made complete when those disabled individuals that would use the end application are tested.

Important to consider are the actual rehabilitation applications that could be developed from the myoelectric control scheme presented. Much of this discussion stems from a qualitative analysis of the experiences occurred during testing of the CCS subject. It would seem that it is possible to use this pattern recognition scheme in single DOF applications for rehabilitation exercises such as those used in physical therapy on a joint by joint basis. The high classification results that occurred using the single DOF controllers for the CCS subject point to the feasibility of using an end application that focuses on one DOF. One could develop a rehabilitation device that could enhance the mobility and range of the elbow, wrist or hand. For instance, the CCS subject compensated wrist pronation and supination by using his biceps. One could use the scheme presented here to recognize the subject's intent for pronation and supination and use this information to assist the subject in his motion. This would increase his ability to perform pronation and supination without using compensatory muscles. The CCS subject even noted that he did not attend physical therapy sessions on the days of performing experiments because of the exercise that the tests provided. Physical therapy applications could be developed with help from an occupational therapist currently assisting the BLL at RIT.

The current platform for testing myoelectric control schemes in the BLL is teleoperation. The results of this work show the feasibility of a teleoperation application. The myoelectric control scheme presented earlier was designed to incorporate EMG signals extracted from isometric contractions for motions occurring below the elbow using the lower arm. In order to create an exoskeleton or a wearable robotic system, one would have to incorporate a control scheme to account for trajectory tracking of the upper arm, as well, where motions requiring multiple degrees of freedom primarily at the elbow and shoulder joint occur simultaneously. A potential platform for achieving this is discussed in the next section which presents the development of a myoelectric control scheme based on a TDNN.

\subsection{Myoelectric control scheme based on a time delayed neural network}

One of the main goals of this work was to study the applicability of the TDNN as a possible myoelectric control scheme for a rehabilitation robot application. The feasibility of this was investigated by analyzing the effect of decreasing the total delay of the TDNN in conjunction with varying other TDNN parameters. $\mathrm{Au}$ and Kirsch [1] had optimal values of $875 \mathrm{~ms}$ and $125 \mathrm{~ms}$ for their total delay and delay interval respectively. That amount of delay is not possible for a real time myoelectric control scheme. The first subject's data was used to determine the optimal parameters for the TDNN and then those optimal parameters were tested on the data from the four remaining subjects.

Based on the first subject's results, it was determined that the number of neurons in the hidden layer did not 
have an effect on the accuracy of the TDNN. So it was decided that a hidden layer of ten should be chosen as the optimal number of neurons. Larger number of neurons that were tested would take up more resources in the end application. In order to view the results in a manner easier to analyze, Table 2 was created. Table 2 presents the results for Subject 1's TDNNs which had ten neurons in the hidden layer. This table shows that the most accurate TDNNs occurred for a TDNN of $300 \mathrm{~ms}$ followed by $600 \mathrm{~ms}$ and $900 \mathrm{~ms}$ respectively. The error of the TDNNs was only slightly different for the total delays of $300 \mathrm{~ms}$ and $600 \mathrm{~ms}$ but was much worse for the $900 \mathrm{~ms}$ total delay. This was a little surprising because the optimal delay for previous work by $\mathrm{Au}$ and Kirsch was $875 \mathrm{~ms}$ which is very close to $900 \mathrm{~ms}$. Also seen in Table 2 is that the time delay intervals using $100 \mathrm{~ms}$ provided for better results than the $50 \mathrm{~ms}$ and $150 \mathrm{~ms}$ intervals. The best accuracy for the TDNNs occurred when using a $300 \mathrm{~ms}$ delay with a $100 \mathrm{~ms}$ delay interval which resulted in an average error of $15.7^{\circ}$. Therefore, the optimal TDNN parameters were chosen to be a total delay of $300 \mathrm{~ms}$, a time delay interval of $100 \mathrm{~ms}$, and a hidden layer of ten neurons. The overall errors in degrees for each subject's TDNN were shown in Table 5. While subject one had the least amount of error with $15.7^{\circ}$, the other subjects had similar results. Subject 2 and Subject 5 had similar errors of $17.0^{\circ}$ and $17.4^{\circ}$ respectively. Subject 4 and Subject 3 were slightly worse with average TDNN errors of $21.5^{\circ}$ and $23.6^{\circ}$ respectively. These errors are similar to the errors reported by $\mathrm{Au}$ and Kirsch which were around $20^{\circ}$. The specific errors in the shoulder and elbow for each test trial are shown in Table 4.

It is difficult to gain a full understanding of the TDNN's ability to predict joint positions by only viewing quantitative data. The TDNN output for each subjects' trials were graphed versus the actual joint position in order to view the results qualitatively. Several observations can be taken away from the graphed results. The TDNN has the ability to predict joint position for multiple DOF at once which is shown in Fig. 12. This is the result during slow reaching for Subject 1. A myoelectric control scheme that could control multiple DOF at once is highly desirable. This would be a benefit that is not offered by the typical myoelectric control scheme based on pattern recognition that was presented in the previous section. Another observation seen from some of the data is the poor accuracy of the TDNN for an immobile DOF while the other DOF is moving. This is shown in Fig. 11 which is the output for Subject 2 during fast shoulder movement. While the TDNN was able to track the shoulder position with great accuracy, the ability of the TDNN to track the stationary elbow joint was poor. Some trials seemed to show that the TDNN had a great ability to predict joint positions from EMG and yet other trials had bad performances. This probably resulted from a disparity between the training data and the testing data. Another important observation to make from the TDNNs is the fact that they map EMG data from dynamic and complex reaching motions. This is another advantage over the Gaussian classifier based myoelectric control scheme approach presented previously which uses isometric EMG data.

The quantitative results showed that decreasing the total delay while maintaining the same level of joint position accuracy was possible. One question that must be answered now is what the actual myoelectric control scheme would look like in real time. Although the suggested TDNN has a $300 \mathrm{~ms}$ delay associated with it, the actual myoelectric control scheme would have a slightly larger delay due to the processing time needed to calculate the TDNN output when fed EMG inputs. The average time needed for the TDNN in the MATLAB environment was $14 \mathrm{~ms}$. Because of this processing delay, a continuous output from the TDNN would not be possible. It would therefore be recommended to give inputs to the TDNN every $15-20 \mathrm{~ms}$ which would adjust for the amount of time needed to process the output. Something else to consider is filtering the output of the TDNN with a low pass filter. Looking at the qualitative results shows that the TDNN output can jump erratically instead of maintaining a smooth output which is desired. A low pass filter would smooth the output and result in a more user friendly control scheme. One must also consider the precision that the TDNNs must have. Across all subjects the average error was $19.0^{\circ}$ which could cause problems if there was a need to be very accurate. Ways at decreasing the error by possibly including kinematic or force data into the TDNN should be considered.

Once this scheme has been implemented in real time, one would want to consider the possible rehabilitation applications that could be developed using this myoelectric control scheme. The goal of the BLL is to develop exoskeleton or orthotic systems which assist those with neuromuscular conditions and diseases. The TDNN approach is advantageous to use because it is based on natural motions of the arm and can con- 
trol multiple DOF at once. Future tests would need to account for a load being used in the trials of training data which would alter the EMG signals.

\section{Conclusion}

This work presented two different myoelectric control techniques for rehabilitation robot applications. The first was a typical approach based on a Gaussian classifier. This myoelectric control scheme combined three DOF (elbow, wrist, and hand) into one controller. It was then tested with ten healthy subjects and achieved an average accuracy of $89.52 \%$. The healthy subjects created a baseline understanding of the myoelectric control scheme which could be modified for someone with a neuromuscular condition. This work also developed a myoelectric control scheme for a subject with CCS. To the authors' knowledge, a myoelectric control scheme has yet to be developed for a subject with CCS. Single DOF controllers were built for the CCS subject to initially gauge the ability of the subject to use a myoelectric control scheme. He achieved $100 \%, 96.30 \%$, and $85.19 \%$ accuracy for the elbow, hand, and wrist controllers respectively. Several adaptations needed to be made to the locations of the electrodes prior to testing the three DOF controller due to the subject using compensatory muscles for certain classes of movement. Once these changes were made, the subject achieved $68.25 \%$ accuracy. Potential rehabilitation applications include physical therapy and teleoperation.

The second myoelectric control scheme was developed to overcome some of the limitations of the first scheme based on pattern recognition. This controller was based on a TDNN and incorporated transient EMG signals that appear during natural reaching motions. An exoskeleton or wearable robot utilizing a TDNN based EMG controller would offer the user movement in more than one DOF simultaneously, which does not exist in current myoelectric control schemes. This work showed that the TDNN accuracy could be maintained as reported in previous work and that the total delay could be decreased from $875 \mathrm{~ms}$ to $300 \mathrm{~ms}$ which is necessary for myoelectric control schemes. The experiments performed to test the TDNNs resulted in an average error of $19^{\circ}$. The next steps in this work would be to implement the TDNN in a real time myoelectric control scheme, as well as explore possibilities of decreasing the error.

\section{Acknowledgements}

This material is based upon work supported by the National Science Foundation under Award No. IIS0748418 and Award No. IIS-0705130.

\section{References}

[1] A.T.C. Au and R.F. Kirsch, EMG-based prediction of shoulder and elbow kinematics in able-bodied and spinal cord injured individuals, IEEE Transactions on Rehabilitation Engineering 8(4) (2000), 471-480.

[2] C.R. Carignan and H.I. Krebs, Telerehabilitation robotics: Bright lights, big future? Journal of Rehabilitation Research and Development 43(5) (2006), 695-710.

[3] G. Cheron, J.P. Draye, M. Bourgeios and G. Libert, A dynamic neural network identification of electromyography and arm trajectory relationship during complex movements, IEEE Transactions on Biomedical Engineering 43(5) (1996), 552-558.

[4] V. Dietz and T. Sinkjaer, Spastic movement disorder: Impaired reflex function and altered muscle mechanics, Lancet Neurol. 6(8) (2007), 725-733.

[5] A.M. Dollar and H. Herr, Lower extremity exoskeletons and active orthoses: Challenges and state-of-the-art, IEEE Transactions on Robotics 24(1) (2008), 144-158.

[6] K. Englehart and B. Hudgins, A robust, real-time control scheme for multifunction myoelectric control, IEEE Transactions on Biomedical Engineering 50(7) (2003), 848-854.

[7] A. Frisoli, F. Salsedo, M. Bergamasco, B. Rossi and M.C. Carboncini, A force-feedback exoskeleton for upper-limb rehabilitation in virtual reality. Applied Bionics and Biomechanics 6(2) (2009), 115-126.

[8] O. Fukuda, T. Tsuji, M. Kaneko and A. Otsuka, A humanassisting manipulator teleoperated by EMG signals and arm motions. IEEE Transactions on Robotics and Automation 19(2) (2003), 210-222.

[9] K.E. Gordon and D.P. Ferris Learning to walk with a robotic ankle exoskeleton, Journal of Biomechanics 40(12) (2007), 2636-2644.

[10] D. Graupe, J. Salahi and K.H. Kohn, Multifunctional prosthesis and orthosis control via microcomputer identification of temporal pattern differences in single-site myoelectric signals, Journal of Biomedical Engineering 4(1) (1982), 17-22.

[11] D. Graupe, J. Salahi and D.S. Zhang, Stochastic analysis of myoelectric temporal signatures for multifunctional single-site activation of prostheses and orthoses, Journal of Biomedical Engineering 7(1) (1985), 18-29.

[12] J. Guest, M.A. Eleraky, P.J. Apostolides, C.A. Dickman and V.K.H. Sonntag, Traumatic central cord syndrome: results of surgical management. Journal of Neurosurgery 97(1) (2002), 25-32.

[13] E. Guizzo and H. Goldstein, The rise of the body bots, IEEE Spectrum 42(10) (2005), 50-56.

[14] A. Gupta, M.K. O’Malley, V. Patoglu and C. Burgar, Design, control and performance of RiceWrist: A force feedback wrist exoskeleton for rehabilitation and training, International Journal of Robotics Research 27(2) (2008), 233-251.

[15] L.J. Hargrove, K. Englehart and B. Hudgins, A comparison of surface and intramuscular myoelectric signal classification, 
IEEE Transactions on Biomedical Engineering 54(5) (2007), 847-853.

[16] G. Hefftner, W. Zucchini and G.G. Jaros, The electromyogram (EMG) as a control signal for functional neuromuscular stimulation. I. Autoregressive modeling as a means of EMG signature discrimination, IEEE Transactions on Biomedical Engineering 35(4) (1988), 230-237.

[17] N. Hogan, H.I. Krebs, J. Charnnarong, P. Srikrishna and A. Sharon, MIT-MANUS - A workstation for manual therapy and training I. IEEE International Workshop on Robot and Human Communication, Tokyo, Japan, September, 1-3, pp. 161-165, 1992.

[18] B. Hudgins, P. Parker and R.N. Scott, A new strategy for multifunction myoelectric control, IEEE Transactions on Biomedical Engineering 40(1) (1993), 82-94.

[19] H.I. Krebs, L. Dipietro, S. Levy-Tzedek, S.E. Fasoli, A. Rykman-Berland, J, Zipse, J.A. Fawcett, J. Stein, H. Poizner, A.C. Lo, B.T. Volpe and N. Hogan, A paradigm shift for rehabilitation robotics. IEEE Engineering in Medicine and Biology Magazine 27(4) (2008), 61-70.

[20] H.I. Krebs and N. Hogan, Therapeutic robotics: A technology push, Proceedings of the IEEE 94(9) (2006), 1727-1738.

[21] H.I. Krebs, B.T. Volpe, D. Williams, J. Celestino, S.K. Charles, D. Lynch and N. Hogan, Robot-aided neurorehabilitation: A robot for wrist rehabilitation, IEEE Transactions on Neural Systems and Rehabilitation Engineering 15(3) (2007), 327-335.

[22] T.A. Kuiken, G.L. Li, B.A. Lock, R.D. Lipschutz, L.A. Miller, K.A. Stubblefield and K.B. Englehart, Targeted muscle reinnervation for real-time myoelectric control of multifunction artificial arms, Journal of the American Medical Association 301(6) (2009), 619-628.

[23] R. Merletti and P. Parker, Electromyography: Physiology, Engineering, and Noninvasive Applications, WileyInterscience, Hoboken, NJ, 2004.

[24] P. Nanda, A. Smith, A. Gebregiorgis, and E.E. Brown Jr, Development of an Upper Extremity Motion Capture System. 2009 IEEE Engineering in Medicine and Biology Conference. Minneapolis, MN. September 1-6, 2009.

[25] T. Nef, G. Marco, and R. Riener, ARMin III - Arm therapy exoskeleton with an ergonomic shoulder actuation, Applied Bionics and Biomechanics 6(2) (2009), 127-142.

[26] Neuronics, 2010. http://www.neuronics.com/cms_en/web/ index.php?id=244\&s=katana.

[27] M.L. Newey, P.K. Sen and R.D. Fraser, The long-term outcome after central cord syndrome - A study of the natural history, Journal of Bone and Joint Surgery-British $\mathbf{8 2 B}(6)$ (2000), 851-855.

[28] M.A. Oskoei, and H.S. Hu, Myoelectric control systemsA survey, Biomedical Signal Processing and Control 2(4) (2007), 275-294.
[29] M.A. Oskoei and H.S. Hu, Support vector machine-based classification scheme for myoelectric control applied to upper limb, IEEE Transactions on Biomedical Engineering 55(8) (2008), 1956-1965.

[30] J.C. Perry, J. Rosen and S. Bums, Upper-limb powered exoskeleton design, IEEE/ASME Transactions on Mechatronics 12(4) (2006), 408-417.

[31] D.M. Rittenhouse, H.A. Abdullah, R.J. Runciman and O. Basir, A neural network model for reconstructing EMG signals from eight shoulder muscles: Consequences for rehabilitation robotics and biofeedback, Journal of Biomechanics 39(10) (2006), 1924-1932.

[32] E.J. Roth, M.H. Lawler and G.M. Yarkony, Traumatic central cord syndrome - Clinical-features and functional outcomes, Archives of Physical Medicine and Rehabilitation 71(1) (1990) 18-23.

[33] R.N. Schmidt, Clinical application driven physiology in biomedical engineering laboratory course education, IEEE Engineering in Medicine and Biology Conference, Shanghai, China, September 1-4, 2005.

[34] C. Skold, R. Levi and S. Ake, Spasticity after traumatic spinal cord injury: Nature, severity, and location, Archives of Physical Medicine and Rehabilitation 80(12) (1999), 1548-1557.

[35] R. Song, K.Y. Tong, X.L. Hu and L. Li, Assistive control system using continuous myoelectric signal in robot-aided arm training for patients after stroke, IEEE Transactions on Neural Systems and Rehabilitation Engineering 16(4) (2008) 371-379.

[36] R. Stiefelhagen, H.K. Ekenel, C. Fugen, P. Gieselmann, H. Holzapfel, F. Kraft, K. Nickel, M. Voit and A. Waibel, Enabling multimodal human-robot interaction for the Karlsruhe humanoid robot, IEEE Transactions on Robotics 23(5) (2007), 840-851.

[37] S. Suryanarayanan and N.P. Reddy, EMG-based interface for position tracking and control in VR environments and teleoperation, Presence-Teleoperators and Virtual Environments 6(3) (1997), 282-291.

[38] S. Theodoridis and K. Koutroumbas, Pattern Recognition, 3rd edn, Academic Press, San Diego, CA, 2006.

[39] A. Tow and K.H. Kong, Central cord syndrome: functional outcome after rehabilitation, Spinal Cord 36(3) (1998), 156-160.

[40] A.J. Woolacott and J.A. Burne, The tonic stretch reflex and spastic hypertonia after spinal cord injury, Experimental Brain Research 174(2) (2006), 386-396.

[41] T. Yamazaki, K. Yanaka, K. Fujita, T. Kamezaki, K. Uemura and T. Nose, Traumatic central cord syndrome: analysis of factors affecting the outcome, Surgical Neurology 63(2) (2005), 95-100. 

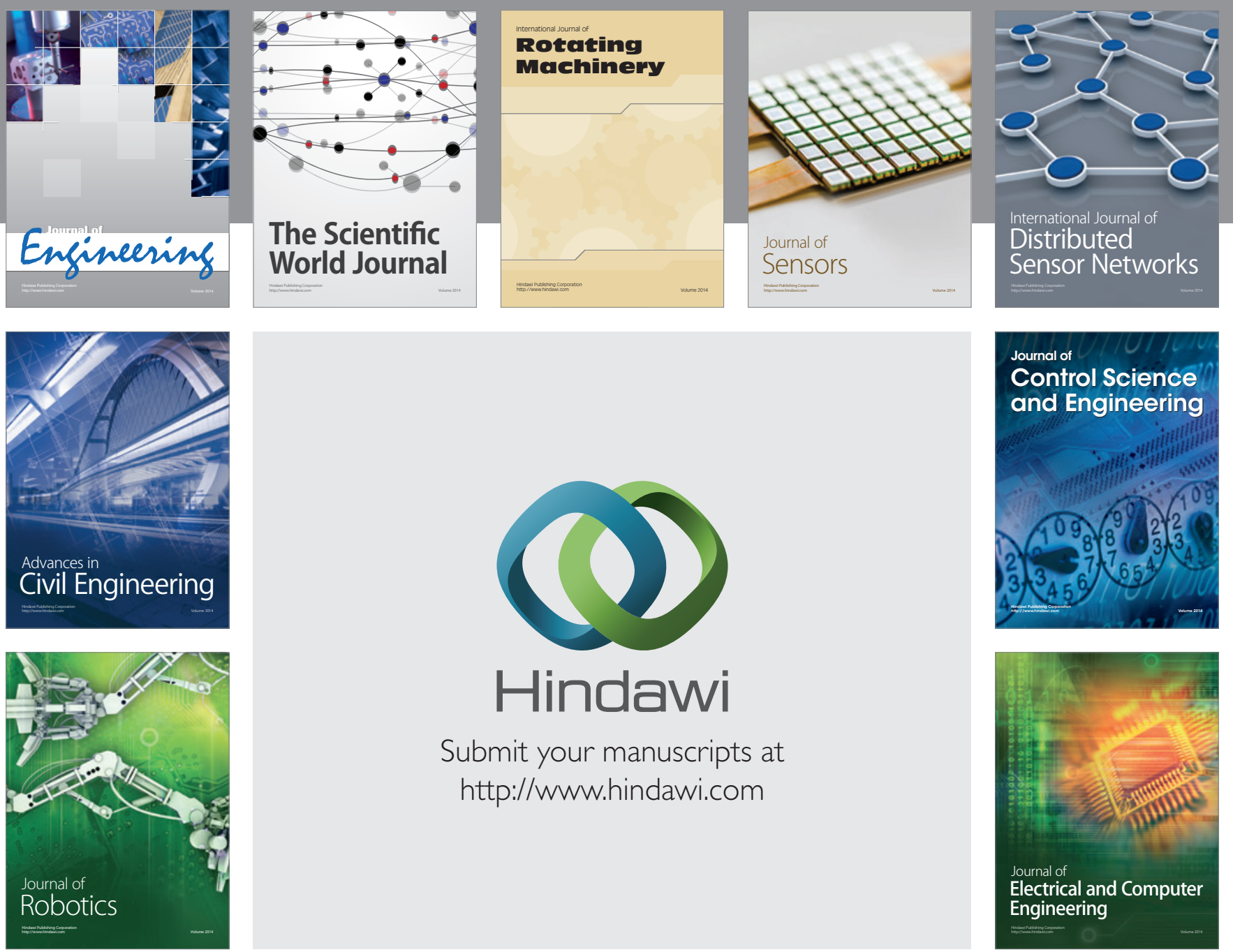

Submit your manuscripts at

http://www.hindawi.com
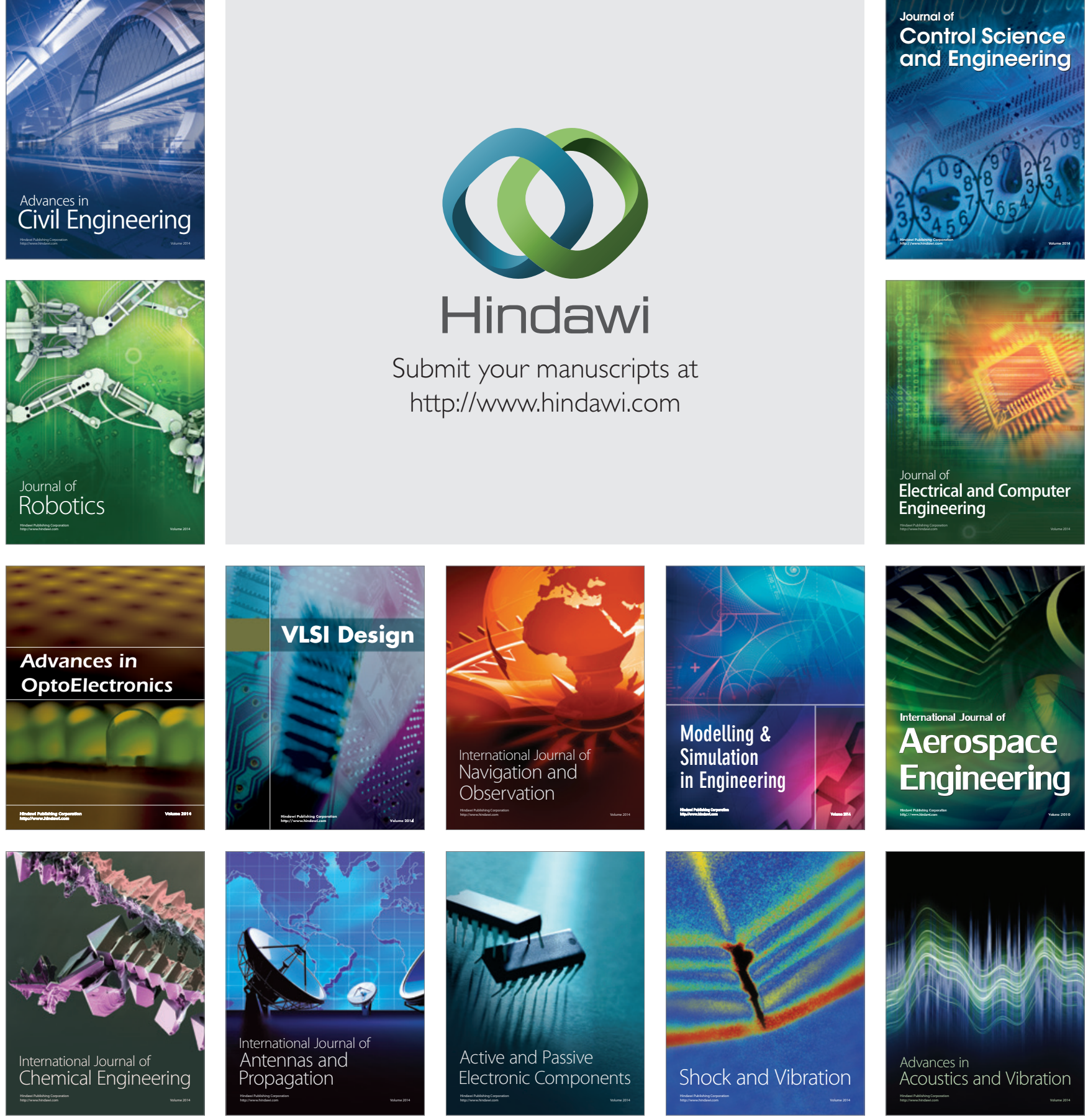